\title{
Human Dimensions of Ecosystem Studies
}

\author{
Charles L. Redman
}

Director of Center for Environmental Studies, Arizona State University, PO Box 873211, Tempe, Arizona 85287-3211,USA

Key words: ecosystems; social science; LTER; urban ecology; interdisciplinary.

Over the course of this century, academic life has progressed in such a manner that scholars who formerly approached inquires from a broad perspective now often constrain themselves within the boundaries of a single discipline. This trend has been a response to the need to effectively engage one's subject matter by developing specialized methodologies, vocabularies, and expectations. Whereas this specialization has proved to be a successful approach for many inquiries, it has also acted to divide scholars in both their training and their investigations. Nowhere has this trend been more pronounced than in environmental sciences whose subject matter naturally divides itself into four domains: biotic, human, geologic, and "built" (Figure 1). Despite well-entrenched disciplinary divisions, most practicing scientists recognize that when treating a comprehensive subject, like human ecosystems, inquiries can not be effectively pursued by specialists working in one of these domains in isolation from the others. For it is by working at the junction of these domains that processes can be best understood and the greatest scientific breakthroughs will be made. Yet, building intellectual bridges between the life, earth, engineering, and social sciences remains extremely difficult. To each of us with our own disciplinary training "human-environmental interactions" has come to mean something quite distinct. For sociologists the "environment" is the social conditions created by humans, for anthropologists the interaction focuses on subsistence pursuits, and for the biologists human participation in an ecosystem is often viewed as a "disturbance." Yet, increasingly people trained in each of these perspectives are recognizing the value-no, the neces-

Received 18 February 1999; accepted 3 March 1999.

E-mail: charles.redman@asu.edu sity—of bridging these disciplinary chasms to create a new means of synthesis. This article reports on the efforts being made by the Central Arizona-Phoenix Long-Term Ecological Research (CAPLTER) project to build these bridges.

In a special section of a recent issue of the journal Science several distinguished ecologists reached the conclusion that "most aspects of the structure and functioning of Earth's ecosystems cannot be understood without accounting for the strong, often dominant influence of humanity" (Vitousek and others 1997, p 494). Furthermore, in a letter to the editor of Science only a few months ago, 20 prominent ecologists called for their colleagues to devote increased energy to understanding and better managing human influences over ecosystem function (Bazzaz and others 1998). Ecologists have long recognized that humans have had significant impacts on their study areas, but these have most often been treated as a form of external disturbance. Although adequate in some respects, it underestimates the pervasiveness and complexity of human influence. To truly understand human actions and influences upon ecosystems, it is essential to thoroughly integrate the approaches developed in the social and economic sciences with those of life and earth scientists.

Recognition that ecosystems are affected by biological, geological (including climate), and human (both social and engineering) forces is a first step in the process of integration. Although most ecologists acknowledge that there are fundamental "drivers" behind biological and geological processes, they have little familiarity with the "drivers" behind human action. Without taking into account these drivers and the interactions they engender, our understanding of ecosystem dynamics both at the 


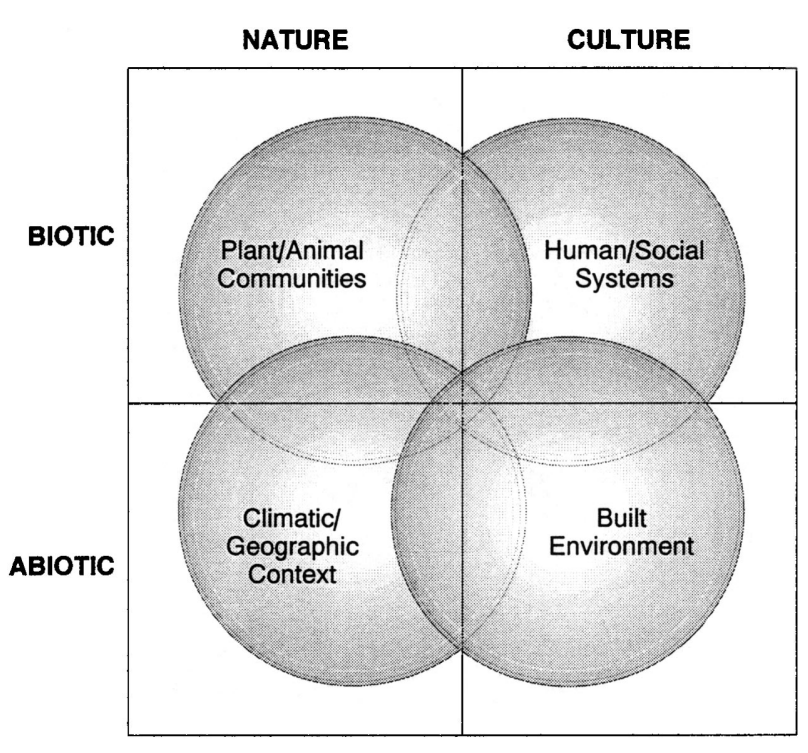

Figure 1. Domains of activity and scientific inquiry within the human ecosystem.

local and global level will be limited, as will be our ability to apply these insights to public policy and land management decisions. By acknowledging the central human component, there comes an added emphasis on new quantitative methods, approaches to modeling, accounting for risk, measuring comparative values, understanding environmental justice, monitoring the effects of innovation and diffusion, and working within a globally interacting network. These added interactions and feedback have traditionally been studied by social scientists in isolation from life and earth scientists because of their own undervaluation of the impact of natural conditions and processes on human decision making [see Costanza (1996) for an alternative viewpoint]. Just as the biologist sees culture as a small and often insignificant influence of nature, the social scientist takes the reverse position that nature has a diminished role in determining human actions (Figure 2). It is time for both camps of researchers to assign appropriate weight to the interdependencies of human ecosystems.

In an attempt to fully incorporate the "human" perspective in the Phoenix and Baltimore LTER projects, several of the principals have suggested a conceptual framework centered on three fundamental drivers of the human components of the ecosystem (Grimm and others, forthcoming):

- flows of information and knowledge, that are guided by the

- incorporation of culturally based attitudes, values, and perceptions, that lead to the

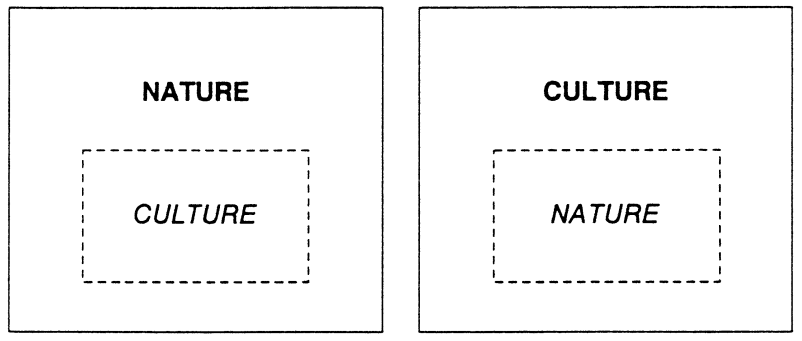

Figure 2. Alternative viewpoints of the relative importance of nature and culture in determining human ecosystem behavior.

- creation and maintenance of institutions and organizations.

These drivers represent processes that condition the enactment of human decisions and subsequent actions. Although understanding the nature and interaction of these drivers must be the ultimate goal of most inquiries, the actual investigations will more often be oriented toward measuring the patterns of behavior these processes create. Defining the following core topics for investigation-each characterized by its activities, structure, and historic trajectory-is key to a comprehensive approach to ecosystem analysis.

\section{- Demographic patterns}

- Economic systems

- Power hierarchies

- Land use and management

- Designed environment

Collectively, these core topics serve as guidelines of inquiry analogous to the five biological core areas identified early in the LTER program's history (primary production, populations, nutrient dynamics, organic matter storage, and disturbance). They reflect the process driving the system, yet at the same time, they are practical guides for field investigation.

To explain causes, predict outcomes, and forecast solutions, human actions must be anchored in time and place-the very context of the landscapes that humans inhabit. Although the entire project is still in a formative stage, social science research has been incorporated into many aspects of the research at the Central Arizona-Phoenix LTER. A study of human valuation as it drives land-use decisions and a second study of environmental risk as it prompts social group formation are underway, but I have chosen to present a brief summary of a third project concerned with human-environmental interactions surrounding urban sprawl. Human-dominated systems, similar to biological systems, undergo continual change that in itself may define key param- 
eters of the ecosystem. At the Central ArizonaPhoenix LTER ever-present urban growth is being used to define a new framework through which to view the ecosystem and to evaluate human actions (Gober and others 1998).

The doubling of population in each of the past 20-year periods has led to a rapid spread of the Phoenix urban area into former farmlands and undisturbed desert landscapes. To monitor this growth, researchers are mapping the locations of new residences in each year of the past decade. The data reveal that almost all new single family residences are along the periphery of the city, and that each year this "doughnut" of new housing moves farther from the urban core. Hence, we find it useful to conceptualize this urban sprawl as a "wave of advance" spreading out from several nodes of urban development. The speed of this wave and its geographic dimensions seem to respond to conditions of the local economy and characteristics of the landscape. In turn, this advance of housing and associated construction transforms the landscape and local ecosystem in what we predict are several regular stages-land surface preparation, infrastructure construction, pioneer housing developments, and filling-in of vacant land with denser housing. Behind the wave, neighborhoods age, leading to a continuing transformational sequence in the nature of human and biotic populations that inhabit those spaces. We argue that virtually every aspect of the ecosystem from soils, to arthropods, to ethnic diversity, to economic investment are fundamentally affected by their position within this transformational sequence. Consequently, we believe that this analysis provides an alternative locational tool to the normal Cartesian grid map of the city that is more sensitive to the key processes that define the urban phenomena.

Although this project was initiated by geographers, it is beginning to attract the participation of scientists whose primary focuses are climate, soils, populations, primary productivity, and nutrient transport, each of whom is in the process of reconceptualizing their approach to work in concert with the social scientists. We are finding the key to interdisciplinary research is that cooperative work will only succeed if the substantive questions each partner is pursuing are enriched by the presence of the other. All too often in interdisciplinary research, one or the other party has been seen as providing a "service" function. That is, one discipline provides "data" to the other without those data really enriching their own inquiries. Even if one accepts Vitousek and others' (1997) assertion that by excluding humans we cannot possibly understand ecosystems, it does not automatically lead to the definition of substantive inquiries of shared interest, or even to mutual respect for the importance of data produced by different disciplines. A century of disciplinary isolation is not easy to overcome, but the potential rewards are great and the alternative of the status quo is unacceptable.

\section{ACKNOWLEDGMENTS}

The initial impetus for this paper came from a meeting of the LTER Network Coordinating Committee, October 1998 in Madison, Wisconsin. Those who contributed to that effort included S. Carpenter, T. Gragson, N. Grimm, M. Grove, C. Harris, T. Kratz, P. Nowak, S. Pickett, and C. Vanderpool. The CAPLTER case study and my own involvement in this work is supported through a grant from the National Science Foundation's Long-term Ecological Research Program (DEB-9714833) codirected by N. B. Grimm.

\section{REFERENCES}

Bazzaz F, and nineteen others. 1998. Ecological science and the human predicament. Science 282:879.

Costanza R. 1996. Ecological economics: reintegrating the study of humans and nature. Ecol Appl 6(4):978-90.

Gober P, Burns EK, Knowles-Yanez K, James J. 1998. Rural to urban land conversion in metropolitan Phoenix, Arizona policy choices. Tempe, AZ: Morrison Institute for Public Policy.

Grimm NB, Grove JM, Pickett ST, Redman CL. Integrated approaches to long-term studies of urban ecological systems. Submitted for publication.

Vitousek PM, Mooney HA, Lubechenco J, Melillo JM. 1997. Human domination of earth's ecosystems. Science 277:494-9. 